\title{
Review: antidepressive agents improve symptoms and reduce pain in patients with functional gastrointestinal disorders
}

Jackson JL, O'Malley PG, Tomkins G, et al. Treatment of functional gastrointestinal disorders with antidepressant medications: a meta-analysis. Am J Med 2000 Jan;108:65-72.

QUESTION: Are antidepressive agents efficacious for treating patients with functional gastrointestinal (Gl) disorders?

\section{Data sources}

Studies were identified by searching Medline (1966-98), PsycLIT (1974-98), EMBASE/Excerpta Medica (197498), the Cochrane Library, and the Federal Research in Progress database using the terms antidepressive agents, serotonin reuptake inhibitors, monoamine oxidase inhibitors, amoxapine, clomipramine, tramipramine, desipramine, doxepin, imipramine, amitriptyline, maprotiline, nortriptyline, protriptyline, trazodone, nefazodone, fluoxetine, fluvoxamine, paroxetine, sertraline, femosetine, venlafaxine, bupropion, citalopram, mianserin, pizotyline, pizotifen, functional colonic diseases, dyspepsia, and abdominal pain. Bibliographies of relevant reviews and studies were scanned.

\section{Study selection}

Randomised controlled trials were selected if they compared an antidepressive agent with placebo in an adult population and outcome data were provided.

\section{Data extraction}

Data were extracted on study quality, setting, country, dose, study duration, follow up, patient numbers and characteristics, comorbid psychiatric disease, adverse effects, outcomes (pain or symptom improvement), and quality indicators.

\section{Main results}

90 citations were reviewed, and 11 met the inclusion criteria. Quality scores were moderate with a mean score of 4 on a 9 point scale. 9 studies evaluated tricyclic antidepressants: amitriptyline and tramipramine in 3 studies, desipramine in 2 studies, and doxepin and clomipramine in 1 study each. 8 studies evaluated the irritable bowel syndrome (IBS), 2 evaluated non-ulcer dyspepsia, and 1 evaluated both disorders.

Treatment with antidepressants improved GI symptoms (table) and reduced pain scores (weighted mean difference $0.9,95 \%$ CI 0.06 to 1.2 ) more than did placebo treatment.

\section{Conclusion}

Antidepressant agents improve symptoms and reduce pain in patients with functional gastrointestinal disorders (the irritable bowel syndrome and non-ulcer dyspepsia).

\section{COMMENTARY}

Medical treatment of functional GI disorders has often been unsatisfactory. This meta-analysis by Jackson $e t$ al presents evidence for the use of antidepressants, but several issues still must be addressed. Meta-analyses may miss unpublished studies that showed no effect; the sensitivity analysis provided suggests that 37 to 157 negative studies would have had to be missed to change the results of the review. In addition, the data found were somewhat limited: Only 8 studies investigated IBS. The others studied non-ulcer dyspepsia, which also seems to respond to antidepressants.

The meta-analysis does not establish whether antidepressants in general are effective. 9 trials used tricyclic antidepressants, and their anticholinergic and analgesic effects might have been more important than their antidepressant effects. The 5HT blocker, mianserin, was effective when given alone (1 study), but trials of serotonin reuptake inhibitors are lacking in patients with IBS.

The role of psychiatric diseases in IBS is complex. A high proportion of patients with IBS are reported to have psychiatric disease, and IBS may be more prevalent in patients with depression. ${ }^{1}$ Patients with IBS and psychological disorders are also more likely to consult their physicians than are patients who have IBS alone. ${ }^{2:}$

Patients with IBS often require medication, which may include tricyclic antidepressants. Placebo responses are high ( $16 \%$ to $59 \%$ in this analysis), and many mild to moderate cases respond to medical attention, including explanation and reassurance. ${ }^{4}$ Despite the high placebo response, however, a trial of antidepressants, especially of a tricyclic antidepressant, in patients with functional GI disorders is justified by the evidence provided by Jackson $e t a l$.

John Calam, MD, MB, ChB Hammersmith Hospital London, UK

1 Lydiard RB, Falsetti SA. Experience with anxiety and depression treatment studies: implications for designing irritable bowel syndrome clinical trials. $A m \quad J$ Med 1999:107(5A):65S-73S.

2 Drossman DA, McKee DC, Sandler RS, et al. Psychosocial factors in the irritable bowel syndrome: a multivariate study of patients and nonpatients with irritable bowel syndrome Gastroenterology 1988;95:701-8.

3 Whitehead WE, Bosmajian L, Zonderman $\mathrm{AB}$, et al Symptoms of psychologic distress associated with irritable bowel syndrome. Gastroenterology 1998;95:709-14.

4 Spiller RC. Problems and challenges in the design of irritable bowel syndrome clinical trials: experience from published trials. Am J Med 1999;107(5A):91S-7S

Symptom improvement with antidepressants $v$ placebo for gastrointestinal disorders with 4 to 12 weeks of treatment*

\begin{tabular}{lllll}
\multirow{2}{*}{$\begin{array}{l}\text { Number of } \\
\text { studies }\end{array}$} & \multicolumn{2}{l}{ Weighted event rates } & & \\
\cline { 2 - 3 } & Antidepressants & Placebo & RBI (95\% CI) & NNT (CI) \\
\hline 11 & $69 \%$ & $36 \%$ & $192 \%(120$ to 300$)$ & $4(3$ to 7$)$ \\
\hline
\end{tabular}

Abbreviations defined in glossary; weighted event rates and RBI supplied by authors.

Source of funding: not stated.

For correspondence: DrJ L Jackson, Department of Medicine-EDP, Uniformed Services University of the Health Sciences, 4301 Jones Bridge Road, Bethesda, MD 20814, USA.Fax +1 202782 7363. 